\title{
NOTES and the mediastinum
}

Author

Institution
Annette Fritscher-Ravens

Experimental Endoscopy Unit, Department of Internal Medicine, University Hospital Schleswig-Holstein, Campus Kiel, Germany
Bibliography

DOI http://dx.doi.org/

10.1055/s-0034-1393132

Published online: 5.11.2015

Endoscopy International Open

2015; 03: E569-E570

(c) Georg Thieme Verlag KG

Stuttgart $\cdot$ New York

E-ISSN 2196-9736

\section{Corresponding author}

\section{Annette Fritscher-Ravens}

Experimental Endoscopy Unit, Department of Internal

Medicine, University Hospital

Schleswig-Holstein

Campus Kiel

24105 Kiel

Germany

fri.rav@btopenworld.com
When natural orifice transluminal endoscopic surgery (NOTES) was introduced more than 10 years ago, it presented the possibility of less-invasive, initially abdominal procedures through the gut wall without skin incision. As procedures were carried out without sufficient basis of experience and knowledge about possible hazards, the most critical points and possible complications of NOTES procedures were defined early on in a white paper [1]. When interest was shown in applying NOTES through the esophagus into the mediastinum [2], it was thought to be an unreasonable and hazardous undertaking. Years earlier, in the mid-1990s, introduction of mediastinal endoscopic ultrasound-fine-needle aspiration (EUS-FNA) caused similar disapproval amongst thoracic surgeons. However, the technique later proved to be a safe and minimally invasive technique that allows complete inspection of and tissue sampling in the entire mediastinum when used in combination with endobronchial ultrasound (EBUS). EUS-FNA has largely replaced standard video-assisted mediastinoscopy (VAM) for evaluation and tissue sampling of malignant and benign mediastinal nodes and lesions.

Evolving experience with NOTES over the last 10 years, since its role in the thoracic and medisastinal space was initially explored, facilitated further understanding of the potential hazards of endoscopic access to the mediastinum. The recent hype around per-oral endoscopic Heller myotomy (POEM), although not strictly NOTES, suggests that a transesophageal approach (to the non-luminal musculature of the esophagus and submucosal tumors through the new "third space", the submucosal tunnel) may become one of the most useful settings for a NOTES-like procedure $[3,4]$. One of the few groups with noteworthy experience with NOTES in the thoracic space has published a study in this issue [5]. In accord with the Noscar White paper, the authors concentrated on feasibility, access, potential hazards, and safety
[1]. Most of the studies available to date were performed in an animal model and only a few in humans, if POEM procedures are excluded [6,7]. This is mainly due to concerns about the safety of the currently available access and closure methods and devices and uncertainty about whether possible complications, including infection and bleeding, can be addressed adequately.

\section{Access/closure \\ $\nabla$}

With the availability of an appropriate tool for closure in 2009/2010, it became possible to perform human studies of NOTES. In 2011, the decision not to market the device brought studies to a halt. For the current study, Cordova's group did not use specific closure tools. Their report clearly showed that submucosal tunneling technique, brilliantly easy to perform with just routinely available tools, did not have much advantage in this setting. Among the 12 animals on which the NOTES procedure was performed, four episodes of bleeding ( $1 \mathrm{fatal}, 3$ manageable) and a burn of the trachea occurred. The authors discuss whether this very high complication rate might have been markedly reduced, had EUS been used for guidance. EUS, however, is not indicated in a setting in which submucosal tunneling is performed simultaneously.

\section{Defects \\ $\checkmark$}

In one third of the animals (4/12), defects of the muscular layer of the esophagus were noted at necropsy. Similar defects were seen in another study, when clip closure was compared to full thickness closure [8]. It is unknown whether later complications are associated with the lack of sufficient wall closure. 


\section{Infection}

$\nabla$

Once in the mediastinum, few further complications were noted, apart from expectable pneumothorax in a few animals. However, post-procedure infection remains one of the main concerns. In Cordova's study, two of 12 animals had mediastinitis. A recent study showed that antibiotics were the single most effective method for avoiding such infection [9] and should be considered routine in such cases. In our mediastinal NOTES studies, antibiotics were given routinely, resulting in a low infection rate in the "healthy animal".

\section{Indications}

$\nabla$

If mediastinal NOTES wants to survive, it is crucial to find useful indications for this more risky procedure. Cordova et al. performed NOTES mediastinoscopy. Because mediastinoscopy can be done safely with EUS plus endobronchial ultrasound without noteworthy complication rates and with excellent accuracy, especially if FNA is added, it is questionable whether that is a suitable indication for NOTES, given the described risk. Rather, it is likely that more invasive procedures not easily done with video-assisted mediastinoscopy might provide more appropriate from a risk-benefit perspective.

The early stir and excitement about NOTES has clearly subsided. Controversy has taken over and most of those who jumped on NOTES in the fast-moving phase have left. The earlier sole and rather ruthless emphasis on "proof of concept" has, in the meanwhile, shifted toward cautious and appropriate work-up and management of complication rates, largely in animals. Progress has been made and some of the initial groups are continuing their work to address the fundamental challenges before clinical use can be debated.

\section{References}

1 ASGE/SAGES Working Group on NOTES White Paper. Gastrointest Endosc 2006; 63: 199-200

2 Fritscher-Ravens $A$, Mosse CA, Ikeda $K$ et al. Endoscopic transgastric lymphadenectomy by using EUS for selection and guidance. Gastrointest Endosc 02 2006; 63: 302 - 306

3 Fritscher-Ravens A, Patel K, Ghanbari A et al. Natural orifice transluminal endoscopic surgery (NOTES) in the mediastinum: long-term survival animal experiments in transesophageal access, including minor surgical procedures. Endoscopy 10 2007; 39: 870-875

4 Makris KI, Rieder E, Swanstrom LL. Natural orifice trans-luminal endoscopic surgery (NOTES) in thoracic surgery. Semin Thorac Cardiovasc Surg 2010; 22: $302-309$

5 Cordova H, Cubas G, Boada M et al. Adverse events of NOTES mediastinoscopy compared to conventional video-assisted mediastinoscopy: a randomized survival study in a porcine model. EIO

6 Hampe J, Schniewind B, Both $M$ et al. Use of a NOTES closure device for full-thickness suturing of a postoperative anastomotic esophageal leakage. Endoscopy 2010; 42: 595-598

7 Hampe J, Schniewind B, Arlt A et al. Closure of anastomotic leakage and iatrogenic perforation with endoscopic suturing: An ongoing pilot study in patients: closure of anastomotic leakage and iatrogenic perforation with endoscopic suturing: An ongoing pilot study in patients. Gastrointestinal Endosopy 2010; 71: AB133

8 Fritscher-Ravens A, Hampe J et al. Clip closure versus endoscopic suturing versus thoracoscopic repair of an iatrogenic esophageal perforation: a randomized, comparative, long-term survival study in a porcine model. Gastrointest Endosc 2010; 72: 1020-1026

9 Ellrichmann M, Dhar S, Sethi A et al. Systematic Evaluation of the Efficacy and Safety of Different Anti-Infective Methods for Transgastric Notes Procedures: A Randomised Controlled Trial in a Porcine Survival Model. Gastrointestinal Endoscopy 2012; 75: AB273 DOI 10.1016/j. gie.2012.03.692 